\title{
Thermoelectric Power of Ion Exchange Membrane Cells Relevant to Reverse Electrodialysis Plants
}

\author{
Kim R. Kristiansen, ${ }^{1, *}$ V. María Barragán, ${ }^{2}$ and Signe Kjelstrup ${ }^{1}$ \\ ${ }^{1}$ PoreLab, Department of Chemistry, Norwegian University of Science and Technology, N-7491 Trondheim, \\ Norway \\ ${ }^{2}$ Department of Structure of Matter, Thermal Physics and Electronics, Complutense University of Madrid, 28040 \\ Madrid, Spain
}

(Received 20 September 2018; revised manuscript received 10 March 2019; published 12 April 2019)

A thermoelectric cell is designed and experiments are carried out in order to measure Seebeck coefficients of ion exchange membranes at different constant concentrations of $\mathrm{NaCl}$ in water. The purpose of the investigation is to explore how a temperature gradient may be applied to increase the efficiency of saline power plants, in particular, of the process of reverse electrodialysis (RED). To evaluate measurements and RED applications, we derive an expression for the thermoelectric potential for a cell with a single membrane and for a RED unit cell. The Seebeck coefficient is interpreted in terms of the Peltier heat of the cell, and further expressed in terms of transported entropies. We find the Seebeck coefficient of the cell, after correcting for temperature polarization, by gradually increasing the membrane thickness. The contribution to the Seebeck coefficient from the membrane varied between 1.41 and $0.98 \mathrm{mV} / \mathrm{K}$ in FUMASEP FKS-PET-75 cation exchange membranes, and between 0.56 and $0.48 \mathrm{mV} / \mathrm{K}$ in FUMASEP FAD-PET-75 anion exchange membranes. The precision in the results is $1 \%$, for $\mathrm{NaCl}$ concentrations between 0.03 and $0.60 \mathrm{~mol} / \mathrm{kg}$. Measurements on the RED unit cell with water samples taken from realistic fresh- and salt-water sources confirmed that a temperature difference has a significant effect, increasing the emf by $1.3 \%$ per kelvin of temperature difference.

DOI: 10.1103/PhysRevApplied.11.044037

\section{INTRODUCTION}

Energy efficiency improvements of industrial processes $[1,2]$ constitute a major way to meet the growing energy demand worldwide. Cullen and Allwood [3] indicate that low-temperature applications of high-grade energy sources, such as fossil fuels, imply losses of around $40 \%-80 \%$ of the potentially useful energy. A substantial fraction $(>60 \%)$ of this turns out as low-grade waste heat at temperatures below $100^{\circ} \mathrm{C}$ [4]. The high-temperature end is well exploited by heat engines, but it is difficult to exploit heat at lower temperatures. Thermoelectric generators are interesting in this context, and the aim of this work is to study the utilization of low-temperature heat sources in thermoelectric energy conversion.

The dominating thermoelectric generators are presently made of solid-state semiconductors. Their efficiencies

*kim.kristiansen@ntnu.no

Published by the American Physical Society under the terms of the Creative Commons Attribution 4.0 International license. Further distribution of this work must maintain attribution to the author(s) and the published article's title, journal citation, and DOI. range from $5 \%$ to $8 \%$ of the Carnot efficiency. Further development of these devices seems restricted by expensive nanoscale engineering [5]. This gives a motivation to explore different materials for thermoelectric energy conversion - ion exchange membranes, see Ref. [6]. Such membranes are already being used to develop saline power plants, like reverse electrodialysis (RED) [7]. In these plants, the Gibbs energy of mixing of sea water and brackish water is exploited in areas such as estuaries, where the two aqueous solutions naturally meet.

The interesting question to pose, is whether the unit cell of the RED plant can benefit from localized heating, thereby adding thermoelectric power to the concentration cell. The aim of this work is to give a quantitative answer to this question. With RED on its path to commercialization, an assessment of its potential for waste-heat harvesting in addition to its standard operation can provide useful input to future engineering solutions. We will study the thermoelectric properties of the membranes systematically as a function of salt concentration. The idea is inspired, e.g., by the work of Kang et al. [8], who investigated the possibility to use thermoelectric energy conversion with high-temperature waste heat in the metallurgical industry in connection with a concentration cell. They observed a 
significant increase in the total potential by combining a molten carbonate concentration cell with a thermoelectric cell.

Much notable experimental work on the thermoelectric potential of cation exchange membranes was done by Tasaka and co-workers before the turn of the century [9-14]. The authors demonstrated that the measured potential depended on the flow rate in their cell, suggesting effects of the diffusion layers adjacent to the membrane. Barragán et al. [15] reported a correction procedure to account for these effects. While the idea to measure thermoelectric potentials of ion exchange membranes is not new [16-18], see Ref. [6] for a detailed review, we present results for other membranes. While it is beneficial to raise the average temperature of the unit cell [18], an effect of a temperature difference in practical applications has not been reported. Temperature polarization, which diminishes the temperature gradient across the system, may have played a role in past experiments. We account properly for polarization effects, using a complete description of the coupled transport phenomena in the cell.

The paper starts with the necessary theoretical description of the experimental cell and the RED cell, before we present the experiments and the results. We conclude with a discussion on how a thermoelectric potential might be used to enhance the concentration cell potential in reverse electrodialysis cells.

\section{THEORY}

We derive a general expression for the emf of a membrane cell with concentration and temperature gradients. Under simplifying conditions, the expression gives the thermoelectric potential that we measure, and the Seebeck coefficient, $\eta_{S}$, from this

$$
\eta_{S}:=\left(\frac{\Delta \phi}{\Delta T}\right)_{j=0} .
$$

Here $\Delta \phi$ is the cell emf, $\Delta T$ is the temperature difference across the cell, and $j$ is the electric current density. The general expression is also used to find the emf of a RED cell in the presence of a temperature gradient. The equation for this cell is used to interpret the measurements with such a cell.

The cell, common to both purposes, has compartments of aqueous $\mathrm{NaCl}$ solutions separated by an ion exchange membrane and $\mathrm{Ag} \mid \mathrm{AgCl}$ electrodes, see Fig. 1. We divide this cell into subsystems that are treated separately, following Kjelstrup and Bedaux [19]. For each subsystem, we identify the local entropy production in terms of the relevant gradients in thermodynamic variables. In the chosen experimental setup, we can neglect effects of viscous flow parallel to the membrane (uniform solutions), and consider the transport problem to be one dimensional. The most important contribution, the membrane contribution, is described first. The contributions from the aqueous solutions, the connecting leads, and the electrode reactions are next added to obtain the total cell potential. The derivation skips some technical steps, which can be found in more detail within the Supplemental Material [20] where relevant.

\section{A. Contribution from the membrane}

Consider the membrane as a planar Gibbs dividing surface, separating two aqueous solutions of $\mathrm{NaCl}$. The temperature $T$, the electrochemical potentials $\tilde{\mu}_{i}$ of the ions $\mathrm{Na}^{+}$and $\mathrm{Cl}^{-}$, and the chemical potential of water vary between the sides. With fluxes of total energy $\left(J_{u}\right)$, ions $\left(J_{i}\right)$, and water $\left(J_{w}\right)$, the entropy production takes the bilinear form [19]

$$
\begin{aligned}
\sigma= & J_{u} \Delta_{\ell, r} \frac{1}{T}-J_{\mathrm{Na}}+\Delta_{\ell, r} \frac{\tilde{\mu}_{\mathrm{Na}^{+}}}{T}-J_{\mathrm{Cl}^{-}} \Delta_{\ell, r} \frac{\tilde{\mu}_{\mathrm{Cl}^{-}}}{T} \\
& -J_{w} \Delta_{\ell, r} \frac{\mu_{w}}{T}
\end{aligned}
$$

with difference notation $\Delta_{n, k} f=f_{k}-f_{n}$. Conventions and nomenclature are taken from Refs. [19,21]. Subscripts $\ell$ and $r$ indicate the left and right compartments, respectively. The electrochemical potentials are $\tilde{\mu}_{i}=\mu_{i}+z_{i} F \psi$, with $\psi$ the electrostatic potential, $F$ the Faraday constant, $z_{i}$ the charge number, and the chemical potential $\mu_{i}=H_{i}-T S_{i}$, with $H_{i}$ and $S_{i}$ the partial molar enthalpy

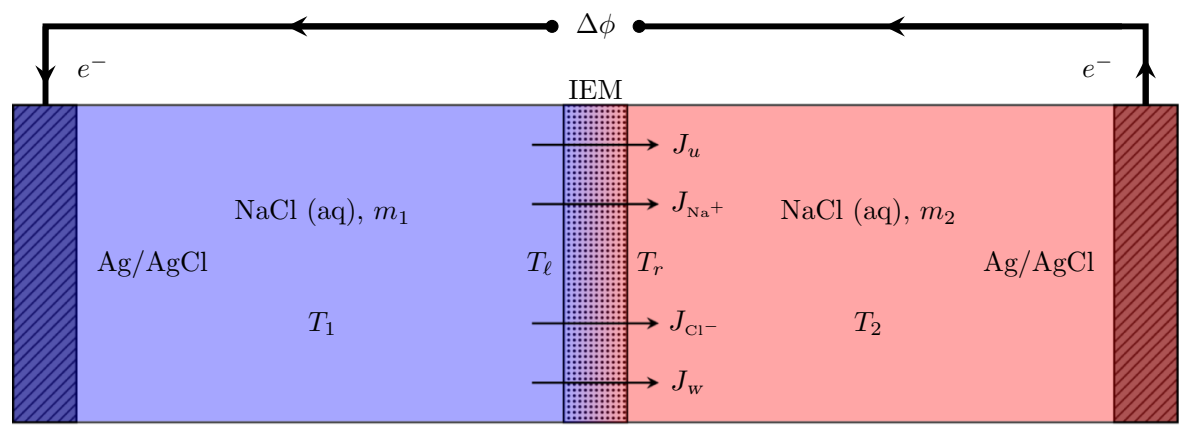

FIG. 1. The single-membrane unit cell considered in this paper. We indicate the energy flux $J_{u}$, and the molar component fluxes $J_{i}$. The positive flux direction is defined to be counterclockwise in this figure. 
and entropy. The entropy production can be rewritten to

$$
\sigma=J_{u} \Delta_{\ell, r} \frac{1}{T}-J_{e} \Delta_{\ell, r} \frac{\mu_{e}}{T}-J_{w} \Delta_{\ell, r} \frac{\mu_{w}}{T}-j \Delta_{\ell, r} \frac{\phi}{T},
$$

with the new variable set

$$
\begin{aligned}
j & =F\left(J_{\mathrm{Na}^{+}}-J_{\mathrm{Cl}^{-}}\right) \\
J_{e} & =c_{-} J_{\mathrm{Na}^{+}}+c_{+} J_{\mathrm{Cl}^{-}} \\
\mu_{e} & =\mu_{\mathrm{Na}^{+}}+\mu_{\mathrm{Cl}^{-}} \\
\phi & =\psi+c_{+} \mu_{\mathrm{Na}^{+}} / F-c_{-} \mu_{\mathrm{Cl}^{-}} / F,
\end{aligned}
$$

with affine coefficients $c_{+}+c_{-}=1$. We have the electric current density $j$, the effective electrolyte flux $J_{e}$, the chemical potential $\mu_{e}$, and the electric potential $\phi$. The coefficients $c_{ \pm}$are determined by the electrode reactions. The electrodes considered here are reversible to the anion, such that the effective electrolyte flux is solely due to the cation flux, giving $c_{-}=1, c_{+}=0$. The electrolyte flux is not a local flux of electrolyte, but measures the net transfer of electrolyte between the two compartments due to a combination of the cation flux and the electrode reactions [22]. The stationary-state energy flux is constant through the membrane

$$
\begin{aligned}
J_{u} & =J_{q}^{\prime \ell}+J_{e} H_{e}^{\ell}+J_{w} H_{w}^{\ell}+j \phi^{\ell} \\
& =J_{q}^{\prime r}+J_{e} H_{e}^{r}+J_{w} H_{w}^{r}+j \phi^{r}
\end{aligned}
$$

with measurable heat flux $J_{q}^{\prime}$, which is the flow of heat that is measurable by a thermometer. The differences in $\mu_{i} / T$ are expanded to linear order in temperature, see Ref. [20]:

$$
\begin{aligned}
\Delta_{\ell, r} \frac{\mu_{i}}{T} & =\frac{1}{T_{r}} \Delta_{\ell, r} \mu_{i}\left(T_{r}\right)+H^{\ell} \Delta_{\ell, r} \frac{1}{T} \\
& =\frac{1}{T_{\ell}} \Delta_{\ell, r} \mu_{i}\left(T_{\ell}\right)+H^{r} \Delta_{\ell, r} \frac{1}{T}
\end{aligned}
$$

and the $H_{i}$ and $\phi$ terms in the energy flux are replaced. Two equivalent forms of $\sigma$ can be obtained:

$$
\begin{aligned}
\sigma & =J_{q}^{\prime r} \Delta_{\ell, r} \frac{1}{T}-J_{e} \frac{\Delta_{\ell, r} \mu_{e}\left(T_{\ell}\right)}{T_{\ell}}-J_{w} \frac{\Delta_{\ell, r} \mu_{w}\left(T_{\ell}\right)}{T_{\ell}}-j \frac{\Delta_{\ell, r} \phi}{T_{\ell}} \\
& =J_{q}^{\prime \ell} \Delta_{\ell, r} \frac{1}{T}-J_{e} \frac{\Delta_{\ell, r} \mu_{e}\left(T_{r}\right)}{T_{r}}-J_{w} \frac{\Delta_{\ell, r} \mu_{w}\left(T_{r}\right)}{T_{r}}-j \frac{\Delta_{\ell, r} \phi}{T_{r}} .
\end{aligned}
$$

We proceed for the moment with the upper form of the entropy production, with reference temperature $T_{\ell}$ and heat flux $J_{q}^{\prime}$. This form of the entropy production leads to the following linear flux-force relations [19]:

$$
\left[\begin{array}{c}
J_{q}^{\prime r} \\
J_{e} \\
J_{w} \\
j
\end{array}\right]=\left[\begin{array}{llll}
L_{q q} & L_{q \pm} & L_{q w} & L_{q \phi} \\
L_{ \pm q} & L_{ \pm \pm} & L_{ \pm w} & L_{ \pm \phi} \\
L_{w q} & L_{w \pm} & L_{w w} & L_{w \phi} \\
L_{\phi q} & L_{\phi \pm} & L_{\phi w} & L_{\phi \phi}
\end{array}\right]\left[\begin{array}{c}
\Delta_{\ell, r}(1 / T) \\
-T_{\ell}^{-1} \Delta_{\ell, r} \mu_{e, T} \\
-T_{\ell}^{-1} \Delta_{\ell, r} \mu_{w, T} \\
-T_{\ell}^{-1} \Delta_{\ell, r} \phi
\end{array}\right]
$$

where the subscripts $T$ of the chemical potentials denote evaluation at the temperature $T_{\ell}$. By solving for the electromotive force $\Delta_{\ell, r} \phi$ across the membrane at open-circuit conditions $(j=0)$, we find

$$
\left(\Delta_{\ell, r} \phi\right)_{j=0}=-\frac{L_{\phi q}}{L_{\phi \phi}} \frac{\Delta_{\ell, r} T}{T_{r}}-\frac{L_{\phi \pm}}{L_{\phi \phi}} \Delta_{\ell, r} \mu_{e, T}-\frac{L_{\phi w}}{L_{\phi \phi}} \Delta_{\ell, r} \mu_{w, T} .
$$

We denote by subscript rev the conditions that $\Delta_{\ell, r} T=0$, $\Delta_{\ell, r} \mu_{e}=0$, and $\Delta_{\ell, r} \mu_{w}=0$. The conductivity matrix $\left\{L_{i j}\right\}$ is symmetric [21], and we identify two of the ratios as [22]

$$
\begin{aligned}
& \frac{L_{\phi \pm}}{L_{\phi \phi}}=\frac{L_{ \pm \phi}}{L_{\phi \phi}}=\left(\frac{J_{e}}{j}\right)_{\mathrm{rev}}=: \frac{t_{\mathrm{Na}^{+}}^{m}}{F} \\
& \frac{L_{\phi w}}{L_{\phi \phi}}=\frac{L_{w \phi}}{L_{\phi \phi}}=\left(\frac{J_{w}}{j}\right)_{\mathrm{rev}}=: \frac{t_{w}}{F} .
\end{aligned}
$$

Here $t_{\mathrm{Na}^{+}}$is the number of moles of electrolyte transported reversibly with the electric current per Faraday of charge. It is equal to the transport number of $\mathrm{Na}^{+}$, defined as the fraction of total charge transfer that is carried by the cation. Likewise, $t_{w}$ is the transference coefficient of water, defined as the number of moles of water transported reversibly with the electric current per Faraday of charge. The last ratio is

$$
\frac{L_{\phi q}}{T_{r} L_{\phi \phi}}=\frac{L_{q \phi}}{T_{r} L_{\phi \phi}}=\frac{1}{T_{r}}\left(\frac{J_{q}^{\prime r}}{j}\right)_{\mathrm{rev}}=: \frac{\Pi^{r}}{T_{r} F},
$$

where we identify the membrane Peltier coefficient $\Pi^{r}$, defined as the measurable heat carried reversibly with the electric current. The left-hand side of Eq. (11) is identified as the Seebeck coefficient

$$
\frac{L_{\phi q}}{T_{r} L_{\phi \phi}}=-\left(\frac{\Delta_{\ell, r} \phi}{\Delta_{\ell, r} T}\right)_{j=0, \Delta \mu_{\mathrm{e}, T}=0, \Delta \mu_{w, T}=0}=:-\eta_{S}^{m, r} .
$$

To proceed, we use the relation between the measurable heat flux and the entropy flux:

$$
T_{r}^{-1} J_{q}^{\prime r}=J_{s}^{r}-S_{e}^{r} J_{e}-S_{w}^{r} J_{w}
$$

with $S_{i}^{r}$ the partial molar entropy of component $i$ evaluated at side $r$. We then express the reversible contribution to the total entropy flux $J_{s}^{r}$ in terms of the transported entropies 
$S_{i}^{*}$ of the ions, in accordance with Refs. [21,23]. Using Eq. (10), we arrive at

$$
\begin{aligned}
\frac{\Pi^{r}}{T_{r}} & =t_{\mathrm{Na}^{+}}^{m} S_{\mathrm{Na}^{+}}^{* r}-t_{\mathrm{Cl}^{-}}^{m} S_{\mathrm{Cl}^{-}}^{* r}-t_{\mathrm{Na}^{+}}^{m} S_{e}^{r}-t_{w} S_{w}^{r} \\
& =t_{\mathrm{Na}^{+}}^{m}\left(S_{\mathrm{Na}^{+}}^{* r}+S_{\mathrm{Cl}^{-}}^{* r}-S_{e}^{r}\right)-S_{\mathrm{Cl}^{-}}^{* r}-t_{w} S_{w}^{r},
\end{aligned}
$$

thus identifying the Seebeck coefficient

$$
\eta_{S}^{m, r}=F^{-1}\left[t_{\mathrm{Na}^{+}}^{m}\left(S_{e}^{r}-S_{\mathrm{Na}^{+}}^{* r}-S_{\mathrm{Cl}^{-}}^{* r}\right)+S_{\mathrm{Cl}^{-}}^{* r}+t_{w} S_{w}^{r}\right]
$$

such that the electromotive force contribution to the cell potential from the membrane is

$$
\Delta_{m} \phi=\eta_{S}^{m, r} \Delta_{\ell, r} T-\frac{t_{\mathrm{Na}^{+}}^{m}}{F} \Delta_{\ell, r} \mu_{e, T}-\frac{t_{w}}{F} \Delta_{\ell, r} \mu_{w, T} .
$$

We now return to the equivalent form of the entropy production with heat flux $J_{q}^{\prime} \ell$ and reference temperature $T_{r}$. The same derivation leads to the expression

$$
\Delta_{m} \phi=\eta_{S}^{m, \ell} \Delta_{\ell, r} T-\frac{t_{\mathrm{Na}^{+}}^{m}}{F} \Delta_{\ell, r} \mu_{\mathrm{e}}\left(T_{r}\right)-\frac{t_{w}}{F} \Delta_{\ell, r} \mu_{w}\left(T_{r}\right),
$$

with the Seebeck coefficient

$$
\eta_{S}^{m, \ell}=F^{-1}\left[t_{\mathrm{Na}^{+}}^{m}\left(S_{e}^{\ell}-S_{\mathrm{Na}^{+}}^{* \ell}-S_{\mathrm{Cl}^{-}}^{* \ell}\right)+S_{\mathrm{Cl}^{-}}^{* \ell}+t_{w} S_{w}^{\ell}\right] .
$$

To linear order in the temperature, we have that

$$
\Delta_{\ell, r} \mu_{i}\left(T_{\ell}\right)+\Delta_{\ell, r} \mu_{i}\left(T_{r}\right)=2 \Delta_{\ell, r} \mu_{i}\left(T_{m}\right)
$$

with $T_{m}=\left(T_{\ell}+T_{r}\right) / 2$ the mean temperature. Identifying the mean Seebeck coefficient $\eta_{S}^{m}=\left(\eta_{S}^{m, \ell}+\eta_{S}^{m, r}\right) / 2$, with the averaged entropies between the two faces of the membrane, we also find

$$
\Delta_{m} \phi=\eta_{S}^{m} \Delta_{\ell, r} T-\frac{t_{\mathrm{Na}^{+}}^{m}}{F} \Delta_{\ell, r} \mu_{e}\left(T_{m}\right)-\frac{t_{w}}{F} \Delta_{\ell, r} \mu_{w}\left(T_{m}\right) .
$$

In the first experiments that we want to describe, this form is advantageous because the two reservoir temperatures are varied in such a way that the average temperature $T_{m}$ is the same for all experiments. Therefore, the chemical potential terms remain unaltered by adjustments to the temperature difference. In the RED cells that are considered, the entropies $S^{\ell}$ and $S^{r}$ can differ substantially, on account of the different salt concentrations.

\section{B. Contribution from aqueous solutions}

The contribution to the Seebeck coefficient of the aqueous solution has a form similar to that of the membrane. The contributions to the emf from the aqueous solutions left and right of the membrane amount to

$$
\Delta_{\mathrm{aq}} \phi=\eta_{S}^{\mathrm{aq}}\left(\Delta_{1,2} T-\Delta_{\ell, r} T\right)-\frac{t_{\mathrm{Na}^{+}}^{\mathrm{aq}}}{F}\left(\Delta_{1,2} \mu_{e, T}-\Delta_{\ell, r} \mu_{e, T}\right) .
$$

The Seebeck coefficient and transport number refer to the two bulk compartments of equal composition. The first term is usually referred to as temperature polarization, while the second term is called concentration polarization. In the experiments, we use a method that eliminates both effects simultaneously, see Sec. II F for details.

\section{Contribution from connecting leads}

When the electrodes are held at different temperatures, there is a net contribution from the electron transport in the temperature gradient across the connecting leads. The external leads are homogeneous conductors $(\mathrm{Cu})$. They connect the platinum wires of the silver electrodes with the potentiometer, where both terminals are at room temperature. The Pt-Cu junctions have, however, the temperatures $T_{1}$ and $T_{2}$, respectively. This gives the contribution

$$
\Delta_{e} \phi=\frac{\Delta S_{e^{-}}^{*}}{F} \Delta_{1,2} T,
$$

where $S_{e^{-}}^{*}$ is the transported entropy of electrons, and $\Delta S_{e^{-}}^{*}=S_{e^{-}}^{*(\mathrm{Cu})}-S_{e^{-}}^{*(\mathrm{Pt})}$ is the difference in the transported entropy between the two different materials.

\section{Contributions from the electrode reactions}

The contributions due to potential changes at the interfaces between electrodes and solutions remain. At the interfaces, the electric current couples with the electrode reactions as the charge carrier is changed across the interface. The electrode reaction is

$$
\mathrm{Ag}+\mathrm{Cl}^{-} \rightarrow \mathrm{AgCl}+e^{-} .
$$

The steady-state entropy production at such an interface can be expressed as

$$
\sigma_{\text {chem }}=-\frac{j}{T}\left(\Delta_{i, o} \phi+\frac{\Delta_{n} G}{F}\right),
$$

where $\Delta_{n} G$ is the Gibbs reaction energy of the neutral components [19]. The flux-force relation close to equilibrium is

$$
j=-\frac{L_{\phi \phi}}{T}\left(\Delta_{i, o} \phi+\frac{\Delta_{n} G}{F}\right),
$$


which means that

$$
\left(\Delta_{i, o} \phi\right)_{j=0}=-\frac{\Delta_{n} G}{F} .
$$

By adding the potential jumps across both electrodesolution interfaces we find, in terms of component entropies

$$
\Delta_{c, 1} \phi+\Delta_{2, a} \phi=\frac{S_{\mathrm{Ag}}-S_{\mathrm{AgCl}}}{F} \Delta_{1,2} T .
$$

This expression is now combined with Eq. (22) to give the contributions to the Seebeck coefficient due to electrode and connecting leads:

$$
\eta_{S}^{\mathrm{el}}=\frac{S_{\mathrm{Ag}}-S_{\mathrm{AgCl}}-\Delta S_{e^{-}}^{*}}{F} .
$$

The corresponding potential jumps at the two electrode interfaces, combined with the potential drops over the electronic leads, is thus

$$
\Delta_{\mathrm{el}} \phi=\eta_{S}^{\mathrm{el}} \Delta_{1,2} T .
$$

\section{E. The total cell potential}

Finally, the total emf of the cell in Fig. 1 is obtained by summing all contributions that we derive. The emf becomes

$$
\Delta \phi=\Delta_{\mathrm{aq}} \phi+\Delta_{m} \phi+\Delta_{\mathrm{el}} \phi
$$

or

$$
\begin{aligned}
\Delta \phi= & \eta_{S}^{\mathrm{el}} \Delta_{1,2} T+\eta_{S}^{m} \Delta_{\ell, r} T+\eta_{S}^{\mathrm{aq}}\left(\Delta_{1,2} T-\Delta_{\ell, r} T\right) \\
& -\frac{t_{\mathrm{Na}^{+}}^{m}}{F} \Delta_{\ell, r} \mu_{\mathrm{e}, T}-\frac{t_{w}}{F} \Delta_{\ell, r} \mu_{w, T} \\
& -\frac{t_{\mathrm{Na}^{+}}^{\mathrm{aq}}}{F}\left(\Delta_{1,2} \mu_{e, T}-\Delta_{\ell, r} \mu_{e, T}\right) .
\end{aligned}
$$

This is the full expression that also applies to half a unit cell of a reverse electrodialysis plant, when the membrane is exposed to solutions of different salinity, as well as to a temperature difference.

The expression will be used to understand the effects of temperature and concentration gradients, as well as temperature- and concentration-polarization effects, on the total cell potential.

When the mean temperature and the concentration is kept constant, we can identify $\eta_{S}^{m}+\eta_{S}^{\mathrm{aq}}$ by plotting the emf vs the temperature difference across the membrane (see below).

To describe a half cell in operation, we add the ohmic resistance drop and the electrode overpotentials. Flux equations must be solved for concentration and temperature profiles.

\section{F. Data-reduction procedure for experiments with single-membrane cells}

When the concentration dependence of $\eta_{S}^{m}$ is solely due to the concentration dependence of the mean partial molar entropies, $\bar{S}_{e}$ and $\bar{S}_{w}$, one can show that [20]

$$
\eta_{S}^{m} \propto-\frac{2 R}{F} \underbrace{\left(t_{\mathrm{Na}^{+}}^{m}-\bar{m} M_{w} t_{w}\right)}_{t_{a}} \ln \left(a_{\mathrm{e}}\right),
$$

where $\bar{m}$ is the mean molality of the electrolyte, $a_{e}$ the electrolyte activity, $M_{w}$ the molar mass of water, and $R$ the universal gas constant. The slope of $\eta_{S}^{m}$ vs $\ln \left(a_{e}\right)$ gives the apparent transport number of $\mathrm{Na}^{+}, t_{a}$. This quantity is often encountered in the literature, see Ref. [24] for a discussion and further references. We use this expression to compare results obtained at different electrolyte concentrations.

Experiments were done with the same electrolyte concentrations in both half cells. In the ideal case, that the concentration- and temperature-polarization effects are zero, Eq. (30) becomes

$$
\Delta \phi=\left(\eta_{S}^{m}+\eta_{S}^{\mathrm{el}}\right) \Delta_{1,2} T .
$$

We compute $\eta_{S}^{\text {el }}$ from data in the literature. From this and the slope of $\Delta \phi$ vs $\Delta_{1,2} T$, we determine $\eta_{S}^{m}$.

Polarization effects are diminished by flowing the solution past the membrane surfaces [15], and by stacking membranes together such that the effective membrane thickness increased.

The results (see below) justified a posteriori that the temperature polarization dominated, so it became sufficient to consider only the temperature profile across the system. We derive in Ref. [20] an expression for the observed Seebeck coefficient as a function of membrane-stack thickness $d_{m}$

$$
\eta_{S}^{\mathrm{obs}}=\frac{\Delta \phi}{\Delta_{1,2} T} \approx \eta_{S}^{\mathrm{el}}+\frac{\eta_{S}^{\mathrm{aq}}}{1+\rho d_{m}}+\frac{\eta_{S}^{m}}{1+1 / \rho d_{m}},
$$

where $\rho$ is a fitting parameter defined in Ref. [20]. As the membrane thickness increases, it follows that a larger proportion of the total temperature difference is maintained across the membrane. The limit values

$$
\begin{aligned}
& \lim _{d_{m} \rightarrow \infty} \eta_{S}^{\mathrm{obs}}=\eta_{S}^{\mathrm{el}}+\eta_{S}^{m} \\
& \lim _{d_{m} \rightarrow 0} \eta_{S}^{\mathrm{obs}}=\eta_{S}^{\mathrm{el}}+\eta_{S}^{\mathrm{aq}}
\end{aligned}
$$

are useful for interpretation of the results.

\section{G. Emf of the nonisothermal reverse electrodialysis cell}

The expression for the single-membrane cell emf can be used to find the emf of a RED power plant. A minimal 


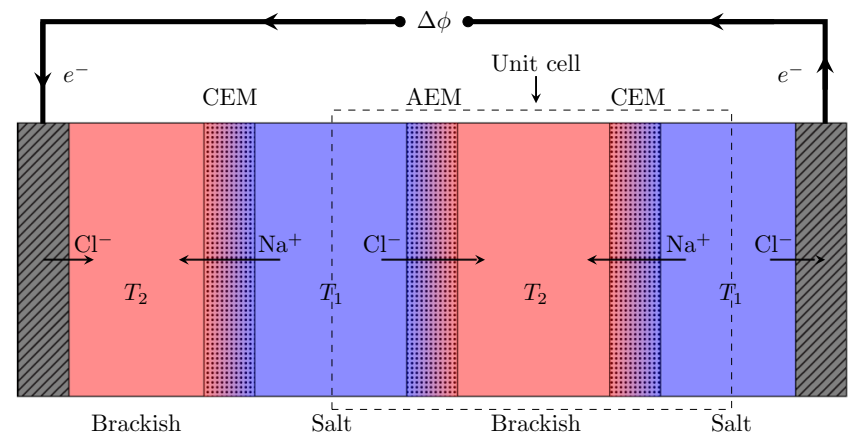

FIG. 2. A minimal reverse electrodialysis cell, with the repeating unit cell indicated by the dashed box.

version of such a plant is sketched in Fig. 2. The RED plant consists of a series of single-membrane cells, and the emf of the RED plant is obtained by adding singlemembrane contributions. The unit cell consists of a pair of cation and anion exchange membranes separating chambers with different salt concentrations. The emf of one unit cell is

$$
\begin{aligned}
\Delta \phi_{\text {unit }}= & \left(\eta_{S}^{\mathrm{AEM}}-\eta_{S}^{\mathrm{CEM}}\right) \Delta_{1,2} T+\frac{t_{\mathrm{Na}^{+}}^{\mathrm{CEM}}-t_{\mathrm{Na}^{+}}^{\mathrm{AEM}}}{F} \Delta_{1,2} \mu_{e, T} \\
& +\frac{t_{w}^{\mathrm{CEM}}-t_{w}^{\mathrm{AEM}}}{F} \Delta_{1,2} \mu_{w, T} .
\end{aligned}
$$

The second term is the primary contribution to the emf. It comes from the Gibbs energy of mixing of the two solutions. The third term is an electroosmotic contribution due to co-transport of water across the membranes. The thermoelectric contribution, which is the focus of this paper, is contained in the first term.

For the sake of completeness, we give the expression for a real RED plant. Let $N$ be the number of unit cells. Assuming that temperature- and concentrationpolarization effects are absent, the emf of the system sketched in Fig. 2 is

$$
\begin{aligned}
\Delta \phi= & -\left(\eta_{S}^{\mathrm{el}}+\eta_{S}^{\mathrm{CEM}}\right) \Delta_{1,2} T \\
& +\frac{t_{\mathrm{Na}^{+}}^{\mathrm{CEM}}}{F} \Delta_{1,2} \mu_{\mathrm{e}, T}+\frac{t_{w}^{\mathrm{CEM}}}{F} \Delta_{1,2} \mu_{w, T} \\
& +N \Delta \phi_{\text {unit }} .
\end{aligned}
$$

When $N=0$, the expression reduces to the expression for a single-membrane cell with a cation exchange membrane (CEM).

\section{EXPERIMENT}

\section{A. Single-membrane experiments}

The thermoelectric cell sketched in Fig. 3 is designed to measure thermoelectric potentials across stacks of ion

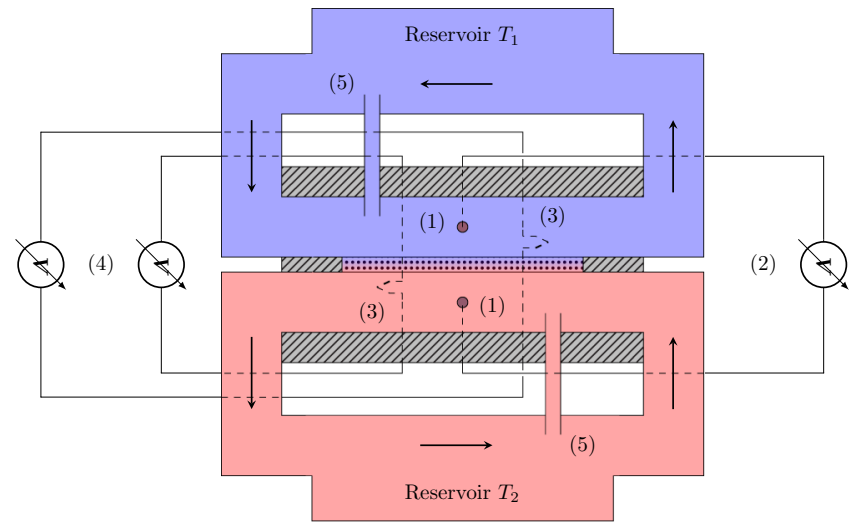

FIG. 3. Sketch of apparatus used for the thermoelectric measurements. Solutions are thermostatted and pumped through the thermocell. Electrodes (1) and thermocouples (3) are indicated on each side of the membrane stack. Back-flow tubing (5) allowed equalization of pressure across the membrane stack.

exchange membranes. The solution flow tangential to the membrane surface is measured to $28 \mathrm{~cm} / \mathrm{s}$, and the exposed membrane area is $8.8 \mathrm{~cm}^{2}$. The temperatures are measured using Omega K-type fine-wire precision thermocouples, and the cell potential is measured with electrodes of chloridized pure silver. Data are acquired and logged using an Agilent 34970A unit with a 34901A module.

The electrode bias is kept in the order $10 \mu \mathrm{V}$, by refreshing the electrodes with new chloride layers whenever the bias exceeded $100 \mu \mathrm{V}$. The salt solutions are prepared using distilled water with conductivity less than $1.5 \mu \mathrm{S} / \mathrm{cm}$. The salt is AnalaR NORMAPUR $\mathrm{NaCl}$ from VWR Chemicals, weighed in all cases to a worst-case accuracy of $0.02 \%$.

The FKS-PET-75 cation exchange membranes (CEM) are delivered by FUMA-Tech in $\mathrm{H}^{+}$form, and the FADPET-75 anion exchange membranes (AEM) in $\mathrm{Cl}^{-}$form. According to specifications, the thickness of each dry membrane sheet is $75 \pm 5 \mu \mathrm{m}$. The CEMs are converted to $\mathrm{Na}^{+}$form by running electric current through the membranes under isothermal conditions at $25^{\circ} \mathrm{C}$. All membranes are further prepared by immersion in the appropriate salt solution, degassed by ultrasound, and kept at $25^{\circ} \mathrm{C}$. For the first week of preparation, the solution is refreshed every 2 days, after which it is refreshed once per week.

The thermoelectric potential is measured by first allowing the system to relax under isothermal conditions, until a stable voltage is read. The reservoir temperatures are then changed such that the temperature difference is \pm 10 $\mathrm{K}$ or $\pm 20 \mathrm{~K}$, while the mean temperature is kept at $25^{\circ} \mathrm{C}$. The measured thermoelectric potential is taken to be the mean voltage over a 15 -min interval of stable readings. The autocorrelation time of the system is estimated to $15 \mathrm{~s}$. In addition to varying the temperature difference, the stack size is varied between 1-20 membranes, and 
the measurements are taken at salt concentrations of 0.03 , 0.10 , and $0.60 \mathrm{~mol} / \mathrm{kg}$.

\section{B. RED unit-cell experiments}

Measurements are carried out on the unit cell indicated in Fig. 2. Water samples are taken from the Nidelven river and the fjord near Trondheim for fresh- and salt-water reservoirs, respectively. The apparatus is identical to that of the single-membrane experiments, but with additional cell elements stacked together with alternating FKS and FAD membranes separating salt and fresh water. The salt water in all compartments is pumped from the same thermostatted reservoir, and the same is done for the fresh water. The electromotive force at open circuit is measured across the unit cell, varying the temperature difference between the two reservoirs. The measurements are carried out under the same conditions as for the single-membrane experiments, and ten membranes are stacked between each cell element.

\section{RESULTS AND DISCUSSION}

\section{A. Single-membrane experiments}

Typical plots of emf vs applied temperature difference are given in Fig. 4. The fact that the lines are straight indicates that there is no significant Thomson effect, which justifies a posteriori the expansion to linear order in the temperature. The observed Seebeck coefficient is taken from the slope of this line.
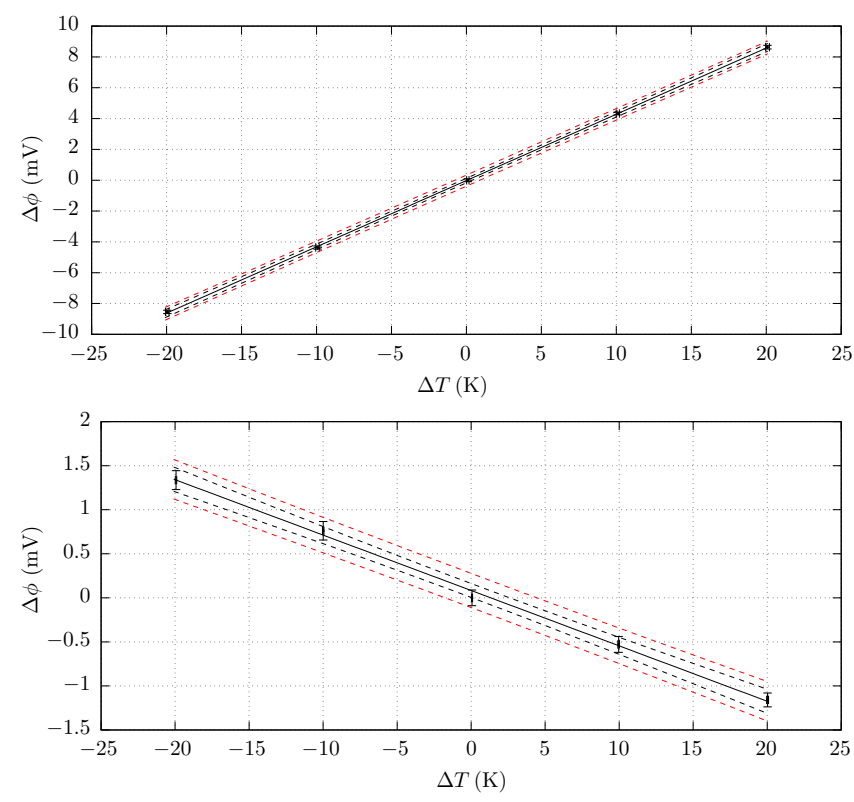

FIG. 4. The observed cell potential $\Delta \phi$ vs the applied temperature difference $\Delta_{1,2} T$ over a stack of 20 FKS-PET-75 (top) and FAD-PET-75 (bottom) membranes in $0.60 \mathrm{~mol} / \mathrm{kg}$ aqueous $\mathrm{NaCl}$ solution. The dashed black and red lines indicate $95 \%$ confidence and prediction, respectively.
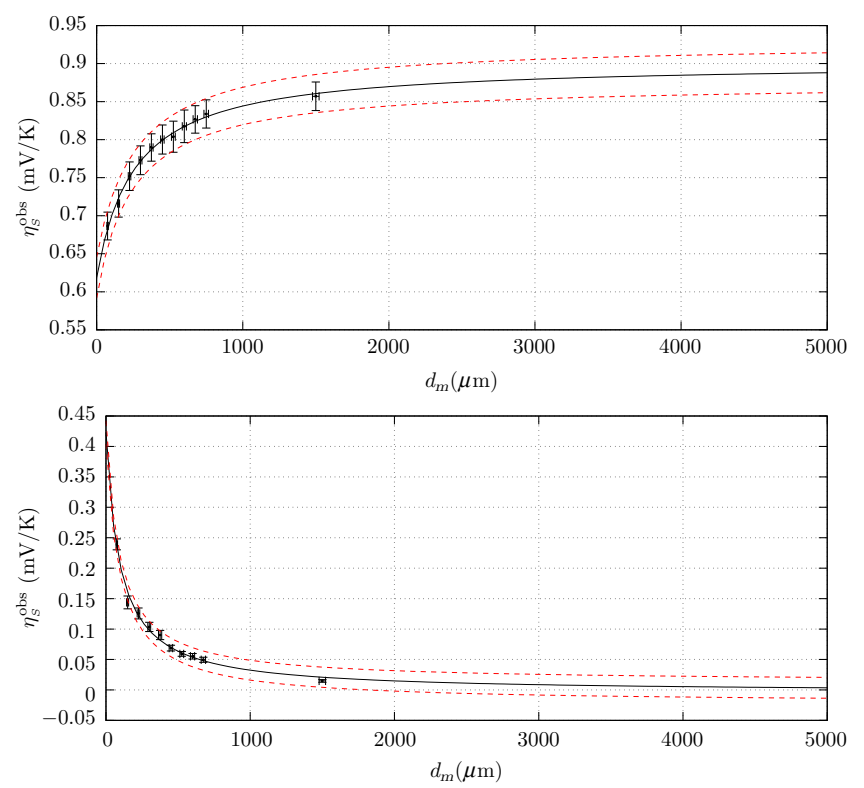

FIG. 5. The observed Seebeck coefficient vs the effective dry thickness of a stack of FKS-PET-75 (top) and FAD-PET-75 (bottom) membranes in $0.03 \mathrm{~mol} / \mathrm{kg}$ aqueous $\mathrm{NaCl}$ solution. The dashed lines indicate $95 \%$ confidence.

The variation in the observed Seebeck coefficient with stack thickness is plotted in Fig. 5, along with the nonlinear least-squares fit of the model in Eq. (33) with confidence intervals acquired by residual resampling [25]. We observe that the model fits the observations, and that a dry-stack thickness of $1.5 \mathrm{~mm}$ gives results close to the limit $d_{m} \rightarrow$ $\infty$ within errors. The same type of convergence is also observed with respect to systematic variation of stirring rate in the work by Barragán et al. [15]. This fortifies our confidence in both methods, that they eliminate the same physical effect.

The contribution to the Seebeck coefficient from the electrodes is calculated, neglecting the difference in transported entropy of electrons across the $\mathrm{Pt}-\mathrm{Cu}$ junctions, and using tabulated values for the entropy of electrode materials [26]. The result is $\eta_{S}^{\mathrm{el}}=-0.545 \mathrm{mV} / \mathrm{K}$. The membrane contributions to the Seebeck coefficient are then calculated according to Eq. (32). We report all calculated membrane contributions, with stack thickness $1.5 \mathrm{~mm}$, in Table I. At $0.03 \mathrm{~mol} / \mathrm{kg}$ salt concentration, the parameter $\rho$ is estimated to $0.004 \mu \mathrm{m}^{-1}$ for the FKS membrane, and 0.011 $\mu \mathrm{m}^{-1}$ for the FAD membrane.

The value in the limit $d_{m} \rightarrow 0$ in Fig. 5 coincides with the value $0.63 \mathrm{mV} / \mathrm{K}$ observed for the aqueous solution of $\mathrm{NaCl}$ at $0.01 \mathrm{~mol} / \mathrm{kg}$ [19]. Since $0<t_{\mathrm{Na}^{+}}^{m}<1$, and $\bar{m} M_{w} \approx 0.01$ at most, we expect $t_{a}$ to be much smaller for the AEM than the CEM. Therefore, the concentration dependence of $\eta_{S}^{m}$, approximated by Eq. (31), is expected to be stronger for the CEM than the AEM. The observed Seebeck coefficient of the membranes can be plotted vs 
TABLE I. Calculated values of $\eta_{S}^{m}$ from observed thermoelectric potentials at different salt concentrations, according to Eq. (32) with $\eta_{S}^{\mathrm{el}}=-0.545 \mathrm{mV} / \mathrm{K}$.

\begin{tabular}{lcc}
\hline \hline $\begin{array}{l}\text { FKS } \\
(\mathrm{mol} / \mathrm{kg})\end{array}$ & $\begin{array}{c}\eta_{S}^{m} \\
(\mathrm{mV} / \mathrm{K})\end{array}$ & $\begin{array}{c}\text { FAD } \\
\eta_{S}^{m} \\
(\mathrm{mV} / \mathrm{K})\end{array}$ \\
\hline 0.03 & $1.41 \pm 0.02$ & $0.560 \pm 0.002$ \\
0.10 & $1.22 \pm 0.02$ & $0.542 \pm 0.002$ \\
0.60 & $0.98 \pm 0.01$ & $0.482 \pm 0.006$ \\
\hline \hline
\end{tabular}

$\ln \left(a_{e}\right)$. Assuming that the transported entropies of the ions are independent of the concentration of electrolyte in external solution, the slope of the line is $-2 \ln (10) R t_{a} / F$, see Fig. 6. Under this assumption, we find $t_{\mathrm{a}}=0.17 \pm 0.07$ for the AEM, and $0.89 \pm 0.05$ for the CEM on average throughout the tested concentration range. Studies made on water transport through these membranes show that $t_{w}<0$ for the AEM, and $t_{w}>0$ for the CEM [24]. This means that water transport will always reduce the absolute value of the apparent transport number of the counter ion in the membrane, and therefore lower the performance of the cell. We have earlier discussed that this may be a point of attention
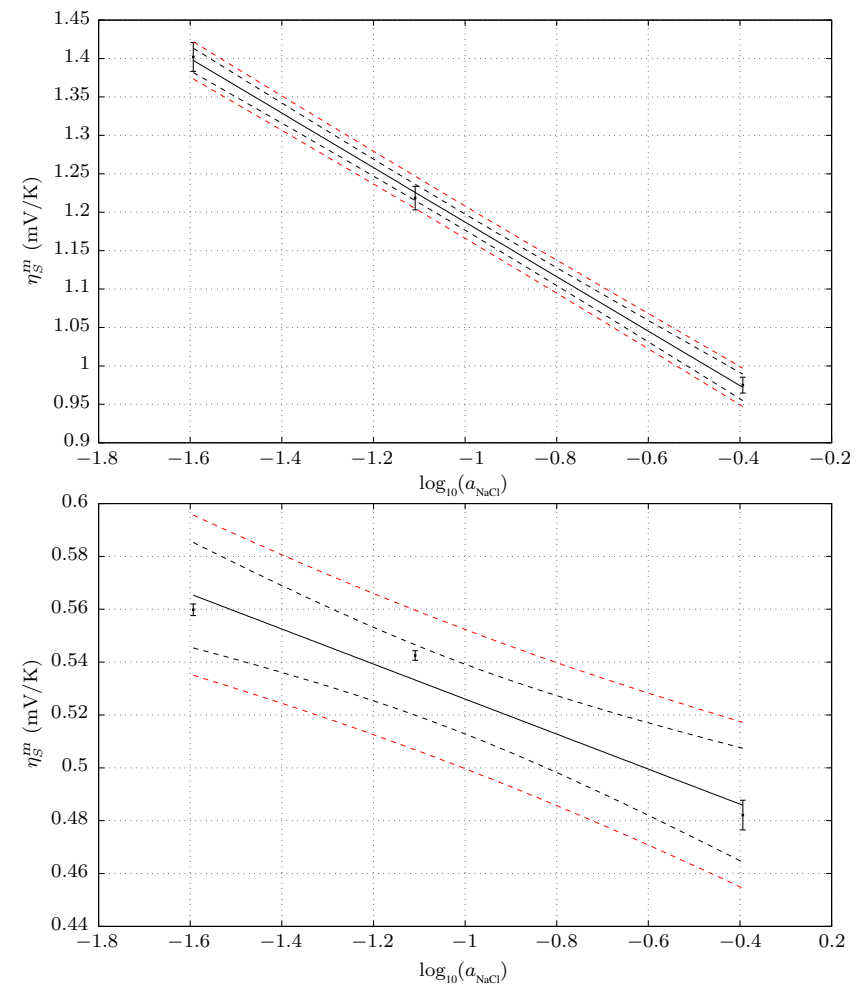

FIG. 6. Plot of the membrane contribution to the Seebeck coefficient of the cell, with 1.5-mm stacks of FKS-PET-75 (top) and FAD-PET-75 (bottom) membranes, against the logarithm of the activity of the external solution. The dashed black and red lines express $95 \%$ confidence and prediction, respectively. for membrane manufacturers [24], the water-transference coefficient is preferably small.

Little is known about the transported entropies of ions in these materials. These quantities express the coupling strength between heat and charge transfer. Knowledge about the quantities allows us to compare thermoelectric properties of different membranes.

From the literature [26], we find $S_{e}=178,160$, and $130 \mathrm{~J} / \mathrm{mol} \mathrm{K}$ for $0.03,0.10$, and $0.60 \mathrm{~mol} / \mathrm{kg}$ solutions, respectively. The sign of $t_{w}$ discussed above means [cf. Eq. (31)] that $t_{\mathrm{Na}^{+}}^{m}<0.17$ for the AEM, and $t_{\mathrm{Na}^{+}}^{m}>0.89$ for the CEM. By assuming that the membranes are perfectly selective, i.e., that $t_{\mathrm{Na}^{+}}^{m}=1$ for CEM and 0 for AEM, we obtain maximum estimates of $S_{\mathrm{Na}^{+}}^{*}+t_{w} \bar{S}_{w}$ for the CEM, and $S_{\mathrm{Cl}^{-}}^{*}-t_{w} \bar{S}_{w}$ for the AEM, see Table II.

The water entropy, $S_{w}^{0}$, is also available from the literature; the standard value at infinite dilution is 69.95 $\mathrm{J} / \mathrm{mol} \mathrm{K}$ [26]. Using activity data from Ref. [27], we calculate the value $70.23 \mathrm{~J} / \mathrm{mol} \mathrm{K}$ at $1 \mathrm{~mol} / \mathrm{kg} \mathrm{NaCl}$, which is a common standard state for electrolyte solutions. With knowledge of the water-transference coefficient, one may check the selectivity assumption, and the calculation of $S_{i}^{*}$. Reliable values of $t_{w}$ is presently missing for the current membranes. Assuming that the ionic transported entropies across the membrane do not change with the concentration of the external solution, the concentration dependence of the values in Table II indicates that $t_{w}$ becomes smaller in magnitude at higher concentrations. The water-transference coefficient should be measured at different salt concentrations in order to investigate this.

In our recent review [6], the second law efficiency of thermoelectric generators was discussed. The figure of merit, $Z T$, can be expressed as

$$
Z T=T_{m} \frac{\kappa \eta_{S}^{2}}{\lambda_{j=0}}
$$

with $\kappa$ the isothermal ionic conductivity, and $\lambda_{j=0}$ the open-circuit thermal conductivity. The figure of merit is positive, and increases monotonously when the entropy production is decreased. Thermal-conductivity data on the presently discussed membranes are not generally available. Burheim et al. [28] measured the thermal conductivity for

TABLE II. Values of the total entropy transported by water and ions in the FKS-PET-75 and FAD-PET-75 membranes, assumed to be perfectly selective.

\begin{tabular}{lcc}
\hline \hline$m$ & FKS & FAD \\
$(\mathrm{mol} / \mathrm{kg})$ & $S_{\mathrm{Na}^{+}+t_{w} \bar{S}_{w}}^{*}$ & $\begin{array}{c}S_{\mathrm{Cl}^{-}}^{*}-t_{w} \bar{S}_{w} \\
(\mathrm{~J} / \mathrm{mol} \mathrm{K})\end{array}$ \\
\hline 0.03 & $42 \pm 2$ & $54.0 \pm 0.2$ \\
0.10 & $42 \pm 2$ & $52.3 \pm 0.2$ \\
0.60 & $35 \pm 1$ & $46.5 \pm 0.6$ \\
\hline \hline
\end{tabular}


a selection of Nafion membranes to about $0.2 \mathrm{~W} / \mathrm{Km}$. This is about a third of the value for water. Using the manufacturer's value of $20 \mathrm{mS} / \mathrm{cm}$ for the ionic conductivity of the FAD membrane, the measured Seebeck coefficients and mean temperature give a $Z T \approx 10^{-3}$, which is a small value. On the other hand, the system is not optimized. Furthermore, the thermal energy is often available, and otherwise wasted. Conductivities and flow-connecting systems should therefore be investigated and optimized for the purpose. A first step in this direction is presented below.

\section{B. RED unit-cell experiments}

A typical measurement series over the RED unit cell is summarized in Fig. 7. The temperature difference $\Delta T$ is defined as the average temperature of the fresh-water cells minus the average temperature of the salt-water cells. The potential across the RED unit cell is given by Eq. (35). As could be predicted from the single-membrane results, the cell emf increases when the fresh water is heated to a higher temperature than the salt water, and is reduced in the opposite case. From three independent measurements, we find an average Seebeck coefficient of $1.9 \pm 0.1$ $\mathrm{mV} / \mathrm{K}$. Added to the isothermal cell emf of approximately $150 \mathrm{mV}$, we find an $1.3 \%$ increase in the cell emf per kelvin of temperature difference used. Therefore, there are two benefits possible by heating the fresh-water source; an increased emf and a reduction in ohmic losses that comes with heating the electrolyte solution [18]. Lowtemperature industrial waste heat may therefore play a role.

The water samples that are used in the experiments are electrolyte mixtures with a large number of salts. Their compositions are analyzed by ICP-MS, and the concentrations of the most abundant nonhalogen elements are shown in Fig. 2 in Ref. [20]. The sodium content of the fresh-water sample is 1 order of magnitude

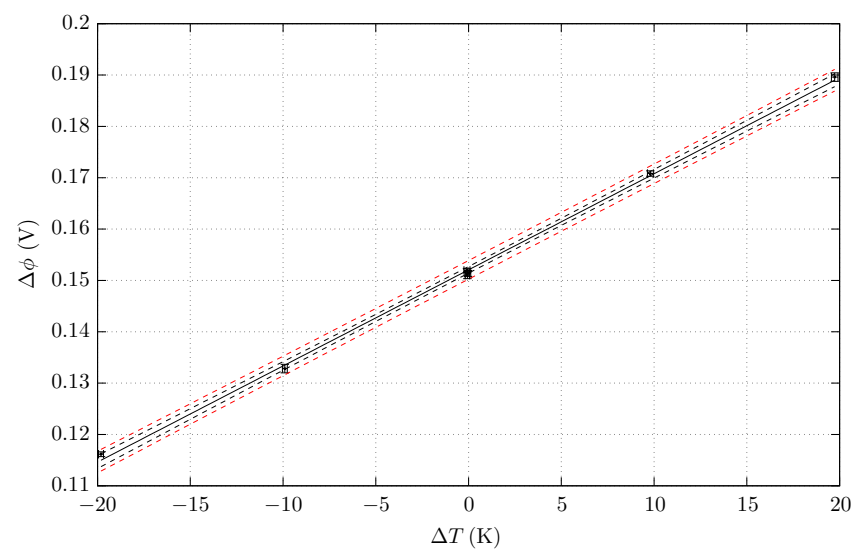

FIG. 7. The measured cell emf $\Delta \phi$, corresponding to $\Delta \phi_{\text {unit }}$ in Eq. (35), across the RED unit cell as the temperature difference $\Delta T$ between the salt and fresh water is varied. smaller than the lowest concentrations considered in the single-membrane experiments. Extrapolation of the singlemembrane results, taking only the effect of sodium into account, yields a predicted Seebeck coefficient near 1 $\mathrm{mV} / \mathrm{K}$ for the concentrations used in the RED cell. This is barely greater than half the measured value. Further work should focus on a systematic exploration of the effect of the most common electrolytes found in the sea-water samples, and also obtain more data with only $\mathrm{NaCl}$ in water, to check whether or not the extrapolation is consistent.

Our results are also relevant for other potential applications. One may consider the application of temperature gradient to also help reduce electrical energy requirements in water purification. This process called electrodialysis, is the reverse of the present one. By reversing the temperature difference, the ideal electric potential required for separation will be smaller.

\section{CONCLUSIONS}

We find that the membranes relevant for RED applications can contribute to the overall cell potential if exposed to a temperature difference. The electrochemical cell potential is measured as a function of the temperature gradient, using $\mathrm{Ag} \mid \mathrm{AgCl}$ electrodes, and the Seebeck coefficient is computed. The experimental values varied between 1.41 and $0.98 \mathrm{mV} / \mathrm{K}$ for the FKS membrane, and between 0.56 and $0.48 \mathrm{mV} / \mathrm{K}$ for the FAD membrane, as the concentration of aqueous $\mathrm{NaCl}$ varied between 0.03 and $0.60 \mathrm{~mol} / \mathrm{kg}$. Counter-ion apparent transport numbers, as determined from the variation in the thermoelectric potential with electrolyte concentration, are $0.89 \pm 0.05$ for FKS, and $0.17 \pm 0.07$ for FAD. These results, combined with the information on the signs of the watertransference coefficients, indicate that both membranes are highly selective. The Seebeck coefficient is interpreted in terms of transported entropies, and the combinations $S_{i}^{*} \pm t_{w} \bar{S}_{w}$ are reported for both membranes under the assumption of perfect selectivity.

The measurements on the RED unit cell confirm that a temperature difference has a significant positive effect, increasing the open-circuit emf by roughly $1.3 \%$ per kelvin of temperature difference. The system is not considered under an operating nonzero current, but the heating of fresh water, which is the part that has the highest ohmic resistance due to low electrolyte concentrations, will serve to reduce the ohmic resistance, and therefore also improve the performance under operating conditions [18]. We conclude that an applied temperature difference is beneficial for reverse electrodialysis.

\section{ACKNOWLEDGMENTS}

The authors are grateful to the Research Council of Norway through its Centers of Excellence funding scheme, 
project number 262644, PoreLab. Anders Granli Haraldsen is thanked for his contribution to the RED cell measurements.

[1] International Energy Agency, Energy Efficiency Indicators (2017).

[2] Study on the Energy Savings Potentials in EU Member States, Candidate Countries and EEA Countries - Final Report (2009).

[3] J. M. Cullen and J. M. Allwood, Theoretical efficiency limits for energy conversion devices, Energy 35, 2059 (2010).

[4] C. Forman, I. K. Muritala, R. Pardemann, and B. Meyer, Estimating the global waste heat potential, Renew. Sust. Energy Rev. 57, 1568 (2016).

[5] Q. Zhang, Y. Sun, W. Xu, and D. Zhu, Organic thermoelectric materials: Emerging green energy materials converting heat to electricity directly and efficiently, Adv. Mater. 26, 6829 (2014).

[6] V. M. Barragán, K. R. Kristiansen, and S. Kjelstrup, Perspectives on thermoelectric energy conversion in ionexchange membranes, Entropy 20, 905 (2018).

[7] J. Moreno, S. Grasman, R. van Engelen, and K. Nijmeijer, Up-scaling reverse electrodialysis, Environ. Sci. Technol. 52, 10856 (2018).

[8] X. Kang, M. T. Børset, O. S. Burheim, G. M. Haarberg, Q. Xu, and S. Kjelstrup, Seebeck coefficients of cells with molten carbonates relevant for the metallurgical industry, Electrochim. Acta 182, 342 (2015).

[9] M. Tasaka, S. Morita, and M. Nagasawa, Membrane potential in nonisothermal systems, J. Phys. Chem. 69, 451 (1965).

[10] M. Tasaka, K. Hanaoka, Y. Kurosawa, and C. Wada, Thermal membrane potential through charfed membranes in electrolyte solutions, Biophys. Chem. 3, 331 (1975).

[11] M. Tasaka, K. Ogawa, and T. Yamazaki, Thermal membrane potential across charged membranes in 2-1 and 1-2 electrolyte solutions, Biophys. Chem. 7, 279 (1978).

[12] K. Hanaoka, R. Kiyono, and M. Tasaka, Thermal membrane potential across anion-exchange membranes in $\mathrm{KCl}$ and $\mathrm{KIO}_{3}$ solutions and the transported entropy of ions, J. Membrane Sci. 82, 255 (1993).

[13] K. Hanaoka, R. Kiyono, and M. Tasaka, Nonisothermal membrane phenomena across perfluorosulfonic acidtype membranes, Flemion S: Part II. Thermal membrane potential and transported entropy of ions, Colloid Polym. Sci. 272, 979 (1994).
[14] R. Kiyono, A. Kuwashita, Y. Tanaka, O. Sekiguchi, and M. Tasaka, Thermal membrane potential acrosspoly(4vinylpyridine.co-styrene) membranes, Bull. Soc. Water Sci. Jpn. 48, 159 (1994).

[15] V. M. Barragán and C. Ruiz-Bauzá, Effect of unstirred solution layers on the thermal membrane potential through cation-exchange membranes, J. Membrane Sci. 125, 219 (1997).

[16] M. Jokinen, J. A. Manzanares, K. Kontturi, and L. Murtomäki, Thermal potential of ion-exchange membranes and its application to thermoelectric power generation, J. Membrane Sci. 499, 234 (2016).

[17] L. Yang, H. Sun, S. Wang, L. Jiang, and G. Sun, A solid state thermogalvanic cell harvesting low-grade thermal energy, Int. J. Hydrogen Energ. 42, 25877 (2017).

[18] A. M. Benneker, T. Rijnaars, R. G. H. Lammertink, and J. A. Wood, Effect of temperature gradients in (reverse) electrodialysis in the ohmic regime, J. Membrane Sci. 548, 421 (2018).

[19] S. Kjelstrup and D. Bedeaux, Non-Equilibrium Thermodynamics of Heterogeneous Systems (World Scientific, Singapore, 2008).

[20] See Supplemental Material at http://link.aps.org/supple mental/10.1103/PhysRevApplied.11.044037 for some of the technical details of the derivations in the theory, which add clarity.

[21] S. R. de Groot and P. Mazur, Non-Equilibrium Thermodynamics (North-Holland Pub. Co., Amsterdam, 1962).

[22] K. S. Førland, T. Førland, and S. Kjelstrup, Irreversible Thermodynamics: Theory and Applications (Wiley, Chichester, 1988).

[23] A. Katchalsky and P. F. Curran, Nonequilibrium Thermodynamics in Biophysics (Harvard University Press, Cambridge, 1967).

[24] A. Zlotorowicz, R. V. Strand, O. S. Burheim, Ø. Wilhelmsen, and S. Kjelstrup, The permselectivity and water transference number of ion exchange membranes in reverse electrodialysis, J. Membrane Sci. 523, 402 (2017).

[25] G. H. Givens and J. A. Hoeting, Computational Statistics (Wiley, Hoboken, 2012), p. 287.

[26] William M. Haynes, CRC Handbook of Chemistry and Physics (CRC Press, Boca Raton, 2015), 96th ed.

[27] R. A. Robinson and R. H. Stokes, Electrolyte Solutions: Second Revised Edition (Dover Publications, London, 2002), p. 476.

[28] O. Burheim, P. J. S. Vie, J. G. Pharoah, and S. Kjelstrup, Ex situ measurements of through-plane thermal conductivities in a polymer electrolyte fuel cell, J. Power Sources 195, 249 (2010). 